in the cells where these receptors are normally expressed (Figure 1). This means that all remaining GABAergic transmission in the $\mathrm{GABA}_{A}$ receptor $\alpha 1$-deficient mouse relies upon a mixture of $\mathrm{GABA}_{A}$ receptors that in many cells are composed of novel $\alpha 2-3 / \beta$, $\beta / \gamma$ subunit combinations. Since these alternative receptors vary in kinetic behavior and pharmacology according to cell type and region, characterizing inhibitory transmission in the mutant nervous system will be a lengthy process, and the design of an effective drug to target this altered pattern may be dependent upon both age and region (21). The authors postulate that the tremor in $\mathrm{GABA}_{\mathrm{A}}$ receptor $\alpha 1^{-/-}$mice may result from a loss of $\mathrm{GABA}_{\mathrm{A}}$ signaling by cerebellar purkinje cells but without actual degeneration of the cells (14). This would be consistent with the pathological studies in ET, which have so far not demonstrated microscopic cerebellar pathology in postmortem brains of patients with ET (22). It should be noted, however, that the $\mathrm{GABA}_{\mathrm{A}}$ receptor $\alpha 1$ mutant mice have an overall reduction in brain size of about $15 \%$, limiting the ability to directly attribute the tremor to this specific purkinje cell-signaling defect.

\section{Conclusions}

In summary, this new model of inherited tremor is welcome because it places $\mathrm{GABA}_{\mathrm{A}}$ receptor $\alpha 1$, a well-known ligand-gated channel subunit, on the list of possible candidate genes for evaluation as a pathogenic cause of human ET and provides an excellent model for tracing the developmental cascade of signaling changes that lead to a tremulous phenotype. If the lessons learned from the investigation of other neurological disorders in mice are any guide, a human variant will soon be in hand; however, whether the human mutant will display tremor is not yet known. Only at that point can we comfortably conclude that the mouse mutant provides a truly "essential" model of the disorder.

Address correspondence to: Joseph Jankovic, Parkinson's Disease Center and Movement Disorders Clinic and Department of Neurology, Baylor College of Medicine, 6550 Fannin, Suite 1801, Houston, Texas 77030, USA. Phone: (713) 798-5998; Fax: (713) 798-6808; E-mail: josephj@bcm.tmc.edu.

1. Dogu, O., et al. 2003. Prevalence of essential tremor: Door-to-door neurologic exams in Mersin Province, Turkey. Neurology. 61:1804-1806.

2. Moghal, S., Rajput, A.H., D’Arcy, C., and Rajput, R. 1994. Prevalence of movement disorders in elderly community residents. Neuroepidemiology. 13:175-178

3. Gulcher, J.R., et al. 1997. Mapping of a familial essential tremor gene, FET1, to chromosome $3 \mathrm{q} 13$. Nat Genet. 17:84-87.

4. Higgins, J., et al. 2004. Haplotype analysis at the ETM2 locus in a Singaporean sample with familial essential tremor. Clin. Genet. 66:353-357.

5. Higgins, J.J., et al. 2005. A variant in the HS1-BP3 gene is associated with familial essential tremor. Neurology. 64:417-421.

6. Jankovic, J. 2002. Essential tremor: a heterogenous disorder. Mov. Disord. 17:638-644

7. Ondo, W.G., et al. 2003. Hearing impairment in essential tremor. Neurology. 61:1093-1097.

8. Deng, H., Le, W., and Jankovic, J. 2005. Parkinson's disease, essential tremor, and premutation alleles of the FMR1 gene. JAMA. In press.

9. Pagan, F.L., Butman, J.A., Dambrosia, J.M., and Hallett, M. 2003. Evaluation of essential tremor with multi-voxel magnetic resonance spectroscopy. Neurology. 60:1344-1347.

10. Ushe, M., et al. 2004. Effect of stimulation frequency on tremor suppression in essential tremor. Mov. Disord. 19:1163-1168.

11. McAuley, J.H. 2001. Does essential tremor originate in the cerebral cortex? Lancet. 357:492-494.

12. Hellwig, B., et al. 2001. Tremor-dominant cortical activity in essential tremor. Lancet. 357:519-523.

13. Wilms, H., Sievers, J., and Deuschl, G. 1999. Animal models of tremor. Mov. Disord. 14:557-571.

14. Kralic, J.E., et al. 2005. Genetic essential tremor in $\gamma$-aminobutyric acid receptor $\alpha 1$ subunit knockout mice. J. Clin. Invest. 115:774-779. doi:10.1172/ JCI200523625.

15. Jankovic, J., Madisetty, J., and Vuong, K.D. 2004. Essential tremor among children. Pediatrics. 114:1203-1205

16. Jankovic, J., et al. 2004. A multicenter, double-blind, placebo-controlled trial of topiramate in essential tremor. Mov. Disord. 19(Suppl. 9):S448-S449.

17. Kash, S.F., et al. 1997. Epilepsy in mice deficient in the $65-\mathrm{kDa}$ isoform of glutamic acid decarboxylase. Proc. Natl. Acad. Sci. U. S. A. 94:14060-14065.

18. Baulac, S., et al. 2001. First genetic evidence of GABA(A) receptor dysfunction in epilepsy: a mutation in the gamma2-subunit gene. Nat. Genet. 28:46-48.

19. Cossette, P., Lortie, A., Vanasse, M., Saint-Hilaire, J.M., and Rouleau, G.A. 2005. Autosomal dominant juvenile myoclonic epilepsy and GABRA1. Adv. Neurol. 95:255-263.

20. Benke, D., et al. 2004. Analysis of the presence and abundance of GABAA receptors containing two different types of alpha subunits in murine brain using point-mutated alpha subunits. J. Biol. Chem. 279:43654-43660.

21. Mohler, H., Fritschy, J.M., Crestani, F., Hensch, T., and Rudolph, U. 2004. Specific GABA(A) circuits in brain development and therapy. Biochem. Pharmacol. 68: $1685-1690$.

22. Rajput, A., Robinson, C.A., and Rajput, A.H. 2004. Essential tremor course and disability: A clinicopathologic study of 20 cases. Neurology. 62:932-936.

\title{
Illuminating the role of type I IFNs in colitis
}

\section{Stefan Wirtz and Markus F. Neurath}

Laboratory of Immunology, I. Medical Clinic, University of Mainz, Mainz, Germany.

\begin{abstract}
Recently, type I interferons IFN- $\alpha$ and IFN- $\beta($ IFN- $\alpha / \beta)$ have been evaluated in pilot clinical trials for the treatment of active ulcerative colitis. However, the underlying mechanisms that may contribute to a potential therapeutic effect are incompletely understood. A new study in this issue demonstrates a protective role for IFN- $\alpha / \beta$, induced by activation of a Toll-like receptor 9-dependent pathway, in a rodent model of experimental colitis (see the related article beginning on page 695).
\end{abstract}

Nonstandard abbreviations used: CPG ODN, CpGcontaining oligodeoxynucleotide; DSS, dextran sulphate sodium; IBD, inflammatory bowel disease; TLR9, Toll-like receptor 9; UC, ulcerative colitis.

Conflict of interest: The authors have declared that no conflict of interest exists.

Citation for this article: J. Clin. Invest. 115:586-588 (2005). doi:10.1172/JCI200524518.
The pathogenesis of Crohn disease and of ulcerative colitis (UC), the 2 major forms of inflammatory bowel disease (IBD), involve a complex interplay among certain genetic, environmental, and immunological factors. Research in the last decade resulted in considerable progress in defining key inflammatory pathways in the inflamed gut and identifying new potential therapeutic targets. In particular, administration or manipulation of immunomodulatory cytokines have been proposed as alternative therapeutic strategies to modulate or inhibit proinflammatory cytokine production in IBD. Although, in the case of Crohn disease, novel strategies to inhibit TNF- $\alpha$ (e.g., administration of the anti-TNF- $\alpha$ monoclonal antibody, infliximab), IFN- $\gamma$, and IL-12 have been used in clinical trials $(1,2)$, relatively few successful studies using anticytokine agents for the treatment of UC have been performed. Recently, type I IFN- $\alpha$ 


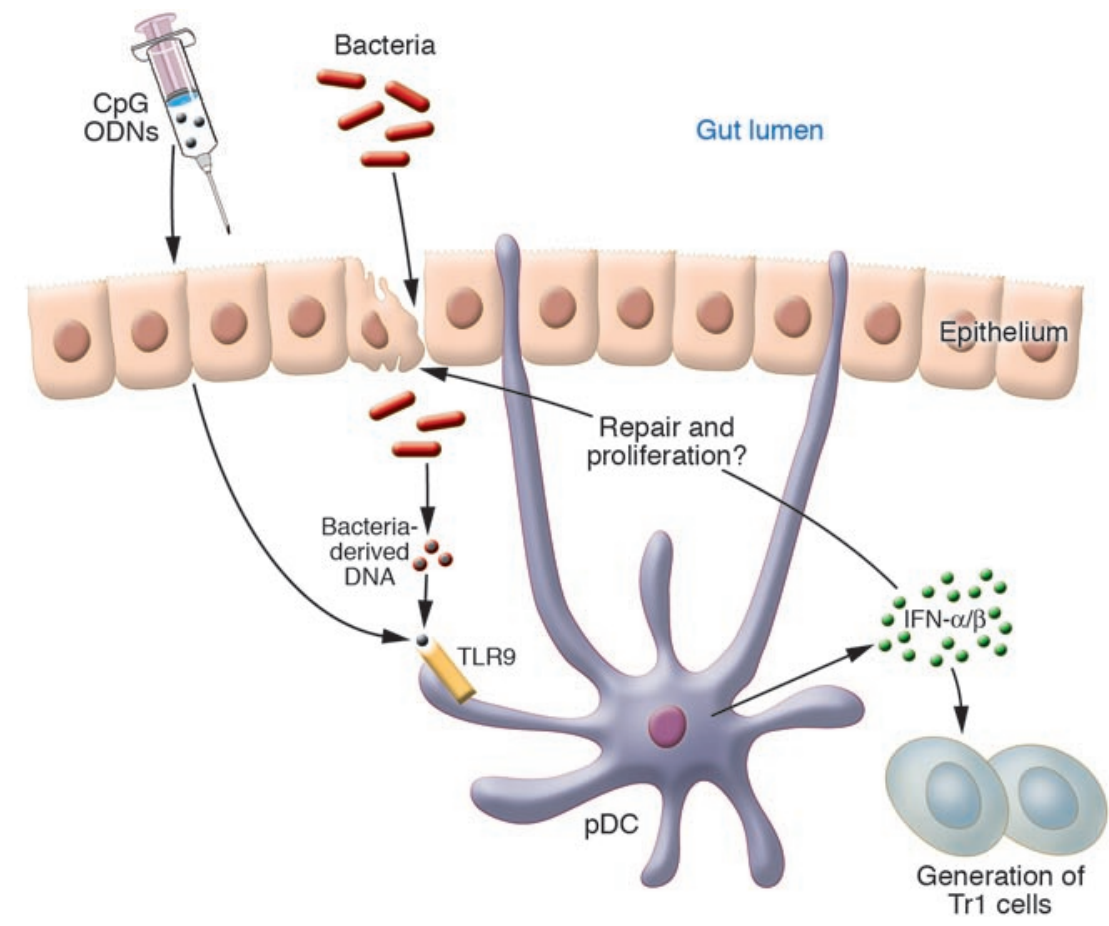

Figure 1

Hypothetical model for a regulatory role of type I IFNs in the gut. Intestinal bacteria entering the mucosa after epithelial damage or exogenous administration of CpG ODNs are recognized by mucosal plasmacytoid dendritic cells ( $p D C s$ ) via TLR9. Stimulated pDCs produce IFN- $\alpha / \beta$, which promote epithelial regeneration or generation of regulatory $T$ cell subsets (e.g., T regulatory 1 cells; $\operatorname{Tr} 1$ ).

and IFN- $\beta$ (IFN- $\alpha / \beta)$ have been evaluated in pilot clinical trials in active UC. In these studies, a subgroup of patients responded to therapy with IFN- $\alpha 2$ a or IFN- $\beta$; however, the results were too preliminary for final conclusions regarding efficacy to be drawn $(3,4)$.

Type I IFNs consist of the protein products of various, mainly intron-less, genes including 14 IFN- $\alpha$ genes and a single IFN- $\beta$ gene. These molecules use a common heterodimeric receptor complex expressed on most cell types throughout the body. Due to their rapid and high level of production following viral infection, they were initially characterized as potent inhibitors of viral replication and hence have been used in the therapy of viral infections such as hepatitis B and C. However, it is now evident that IFN- $\alpha / \beta$ have important immunoregulatory functions, e.g., during inflammation or nonviral infections (5).

\section{The role of IFN- $\alpha / \beta$ in the normal and inflamed gut}

It is astonishing to realize that in spite of the existence of clinical trials on the use of IFN- $\alpha / \beta$ in the treatment of UC, there is only very limited information about their expression and biological function in the immune system of the human gut. Moreover, there is little published data regarding the activity of these molecules in animal models of IBD. In this issue of the JCI, Katakura and colleagues report that they have discovered a protective role for IFN- $\alpha / \beta$ in a murine model of experimental colitis (6). These results underscore a potentially important protective role for type I IFNs in intestinal homeostasis and suggest that strategies to modulate innate immunity may be of therapeutic value for intestinal inflammatory conditions.

In previous reports, it was shown somewhat unexpectedly by the Katakura group and others that pretreatment of mice, before induction of dextran sulphate sodium (DSS) colitis with bacterial DNA or synthetic oligonucleotides containing unmethylated CPG dinucleotides, ameliorates colonic inflammation (7-9). Based on these earlier observations, Katakura et al. (6) have now explored the role of IFN- $\alpha / \beta$, which are strongly induced by $\mathrm{CpG}$-containing oligodeoxynucleotides (CPG ODNs), in CD $11^{\text {low }} \mathrm{B} 220^{+} \mathrm{Gr} 1^{+}$plasmacytoid dendritic cells and macrophages in acute DSS-induced colitis in mice (Figure 1). They demonstrate in several experiments the existence of a Tolllike receptor 9-dependent (TLR9-dependent) mechanism of IFN- $\alpha / \beta$ induction, which accounts for $\mathrm{CPG} O \mathrm{ODN}$-mediated protection. This effect is also evident in mice deficient in T and B lymphocytes and, hence, apparently independent of the presence of the adaptive immune system. Mice lacking the IFN- $\alpha / \beta$ receptor were resistant to the CpG ODN-mediated effect and, interestingly, in comparison to wild-type controls, these mice suffered from increased mortality rates in response to DSS treatment without CpG ODN pretreatment. This suggests that endogenous mechanisms, such as the entry of bacterial DNA into the mucosa after DSSinduced epithelial damage, induce production of antiinflammatory proteins such as IFN- $\alpha / \beta$. These data now provide a rational basis for an in-depth analysis of IFN- $\alpha / \beta$ function in gut homeostasis. In this regard, real-time PCR experiments with specific IFN primer sets and analysis of the kinetics of the differential expression of IFN response genes could be helpful in the search for an optimal IFN-based therapy. However, there are potential concerns about the therapeutic window of CpG ODN or recombinant IFN- $\alpha / \beta$ $(\mathrm{rIFN}-\alpha / \beta)$ treatment. There is evidence that CpG ODN treatment is only effective when given prior to DSS treatment while administration to mice with chronic colitis in fact worsens disease $(8,10)$. Thus, administration of CpG ODNs in chronically active UC may augment rather than suppress intestinal inflammation. In addition to IFN- $\alpha / \beta$, CPG ODN-TLR9 signaling strongly induces the NF-кB-dependent expression of proinflammatory cytokines, such as IL- 6 and IL-12, that exert potent proinflammatory effects in T cell-dependent colitis $(11,12)$. It will therefore be interesting to see whether rIFN- $\alpha / \beta$ administration has fewer of these unwanted side effects compared to $\mathrm{CpG}$ ODN administration and whether it will be effective in the chronic phase of DSSinduced colitis.

It is well established that the adaptive immune system (in particular $\mathrm{CD}^{+} \mathrm{T}$ cells) is absolutely required for both gut homeostasis and a deregulated chronic immune response against the commensal microflora (13). Although the function of type I IFNs on T cells is incompletely understood, an important rationale for the trial of IFN treatment for UC was the known function of IFN- $\alpha / \beta$ on T cells. In fact, IFN- $\alpha / \beta$ was shown to stimulate production of the antiinflammatory cytokine IL-10 by human 
$\mathrm{CD}^{+} \mathrm{T}$ cells (14). Furthermore, IFN- $\alpha / \beta$ modulates Th1 responses (15) and inhibits production of Th2 cytokines (16), some of which (e.g., IL-5, IL-13) are upregulated in the mucosa of patients with UC (17). Interestingly, recent studies suggest that IFN- $\alpha / \beta$ is able to induce regulatory T cells (18) and, moreover, intestinal $\mathrm{CD}^{+}$plasmacytoid dendritic cells producing IFN- $\alpha / \beta$ after $C p G$ ODN stimulation were shown to induce the differentiation of naive $\mathrm{T}$ cells to IL-10secreting T regulatory 1-like cells (19).

The protective effect of $\mathrm{CpG}$ ODNs seems to be independent of IL-10, as they ameliorate spontaneous colitis in IL-10-deficient mice (7). However, a key remaining question is whether IFN- $\alpha / \beta$ treatment is beneficial in T cell-dependent animal models of colitis such as the well-established adoptive $\mathrm{CD}^{+}$CD45RB ${ }^{\text {high }}$ transfer model (20). Such detailed characterization of the biological function of IFN- $\alpha / \beta$ in experimental animal models will undoubtedly be of great value for future clinical trials in IBD.

Address correspondence to: Markus F. Neurath, Laboratory of Immunology, First Medical Clinic, University of Mainz, Langenbeckstrasse 1, 55101 Mainz, Germany. Phone: 49-6131-172374; Fax: 49-6131-39175508; E-mail: neurath@1-med.klinik.uni-mainz.de.
1. Targan, S.R., et al. 1997. A short-term study of chimeric monoclonal antibody cA2 to tumor necrosis factor alpha for Crohn's disease. Crohn's Disease cA2 Study Group. N. Engl. J. Med. 337:1029-1035.

2. Mannon, P.J., et al. 2004. Anti-interleukin-12 antibody for active Crohn's disease. N. Engl. J. Med. 351:2069-2079.

3. Madsen, S.M., et al. 2001. An open-labeled, randomized study comparing systemic interferon-alpha$2 \mathrm{~A}$ and prednisolone enemas in the treatment of left-sided ulcerative colitis. Am. J. Gastroenterol. 96:1807-1815

4. Nikolaus, S., et al. 2003. Interferon beta-1a in ulcerative colitis: a placebo controlled, randomised, dose escalating study. Gut. 52:1286-1290.

5. Bogdan, C., Mattner, J., and Schleicher, U. 2004. The role of type I interferons in non-viral infections. Immunol. Rev. 202:33-48.

6. Katakura, K., et al. 2005. Toll-like receptor 9induced type 1 IFN protects mice from experimental colitis. J. Clin. Invest. 115:695-702. doi:10.1172/ JCI200522996.

7. Rachmilewitz, D., et al. 2002. Immunostimulatory DNA ameliorates experimental and spontaneous murine colitis. Gastroenterology. 122:1428-1441.

8. Obermeier, F., et al. 2003. Contrasting activity of cytosin-guanosin dinucleotide oligonucleotides in mice with experimental colitis. Clin. Exp. Immunol. 134:217-224.

9. Rachmilewitz, D., et al. 2004. Toll-like receptor 9 signaling mediates the anti-inflammatory effects of probiotics in murine experimental colitis. Gas troenterology. 126:520-528.

10. Obermeier, F., et al. 2002. CpG motifs of bacterial DNA exacerbate colitis of dextran sulfate sodiumtreated mice. Eur. J. Immunol. 32:2084-2092.

11. Atreya, R., et al. 2000. Blockade of interleukin 6 trans signaling suppresses T-cell resistance against apoptosis in chronic intestinal inflammation: evidence in crohn disease and experimental colitis in vivo. Nat. Med. 6:583-588.

12. Simpson, S.J., et al. 1998. T cell-mediated pathology in two models of experimental colitis depends predominantly on the interleukin 12/signal transducer and activator of transcription (Stat)-4 pathway, but is not conditional on interferon gamma expression by T cells. J. Exp. Med. 187:1225-1234.

13. Wirtz, S., and Neurath, M.F. 2000. Animal models of intestinal inflammation: new insights into the molecular pathogenesis and immunotherapy of inflammatory bowel disease. Int. J. Colorectal Dis. 15:144-160.

14. Aman, M.J., et al. 1996. Interferon-alpha stimulates production of interleukin-10 in activated CD4+ T cells and monocytes. Blood. 87:4731-4736.

15. Nguyen, K.B., et al. 2000. Interferon alpha/beta-mediated inhibition and promotion of interferon gamma: STAT1 resolves a paradox. Nat. Immunol. 1:70-76.

16. Kaser, A., Molnar, C., and Tilg, H. 1998. Differential regulation of interleukin 4 and interleukin 13 production by interferon alpha. Cytokine. 10:75-81.

17. Fuss, I.J., et al. 1996. Disparate CD4+ lamina propria (LP) lymphokine secretion profiles in inflammatory bowel disease. Crohn's disease LP cells manifest increased secretion of IFN-gamma, whereas ulcerative colitis LP cells manifest increased secretion of IL-5. J. Immunol. 157:1261-1270.

18. Levings, M.K., et al. 2001. IFN-alpha and IL-10 induce the differentiation of human type $1 \mathrm{~T}$ regulatory cells. J. Immunol. 166:5530-5539.

19. Bilsborough, J., George, T.C., Norment, A., and Viney, J.L. 2003. Mucosal CD8alpha+ DC, with a plasmacytoid phenotype, induce differentiation and support function of $\mathrm{T}$ cells with regulatory properties. Immunology. 108:481-492.

20. Powrie, F., Correa-Oliveira, R., Mauze, S., and Coffman, R.L. 1994. Regulatory interactions between CD45RBhigh and CD45RBlow CD4+ T cells are important for the balance between protective and pathogenic cell-mediated immunity. J. Exp. Med. 179:589-600.

\title{
Vascular remodeling and the kallikrein-kinin system
}

\author{
Oscar A. Carretero
}

Hypertension and Vascular Research Division, Henry Ford Hospital, Detroit, Michigan, USA.

\begin{abstract}
Remodeling of the arterial wall occurs mainly as a consequence of increased wall stress caused by hypertension. In this issue of the JCI, Azizi et al. report that in humans with a kallikrein gene polymorphism that lowers kallikrein activity, the brachial artery undergoes eutrophic inward remodeling in the absence of hypertension or other hemodynamic changes (see the related article beginning on page 780 ). It has also been reported that alterations of the kallikrein-kinin system are associated with formation of aortic aneurysms. Conversely, after vascular injury, kinins mediate the beneficial effect of angiotensin-converting enzyme inhibitors that prevent neointima formation. These findings raise the intriguing possibility that decreased kallikrein-kinin system activity may play an important role in the pathogenesis of vascular remodeling and disease, while increased activity may have a beneficial effect.
\end{abstract}

Conflict of interest: The author has declared that no conflict of interest exists.

Citation for this article: J. Clin. Invest. 115:588-591 (2005). doi:10.1172/JCI200524567.

\section{Kallikrein-kinin system, vascular expression, and release}

Kinins (bradykinin and lysyl-bradykinin) have been implicated in the regulation of renal function, blood flow, and blood pressure (1). The vasodilator, natriuretic, and diuretic effects of kinins are mediated by the release of autacoids such as eicosanoids, NO, and endotheliumderived hyperpolarizing factor. Kinins are released from kininogen by proteolytic enzymes known as kininogenases, the main kininogenases being plasma and tissue (glandular) kallikrein (hereafter referred to as kallikrein). Kinins are rapidly hydrolyzed by a group of peptidases known as kininases (Figure 1). In the blood, kinins circulate in the low picogram range (2-20 pg), a concentration 100 times lower than that needed to decrease blood pressure (2). Thus, if kinins participate in the regulation of blood flow, 